\title{
Experimental test of Mermin inequalities on a five-qubit quantum computer
}

\author{
Daniel Alsina and José Ignacio Latorre \\ Departament Física Quàntica i Astrofísica, Universitat de Barcelona, Diagonal 645, 08028 Barcelona, Spain \\ and Institut de Ciències del Cosmos (ICCUB), Martí i Franquès 1, 08028 Barcelona, Spain
}

(Received 25 May 2016; published 11 July 2016)

\begin{abstract}
Violation of Mermin inequalities is tested on the five-qubit IBM quantum computer. For three, four, and five parties, quantum states that violate the corresponding Mermin inequalities are constructed using quantum circuits on superconducting qubits. Measurements on different bases are included as additional final gates in the circuits. The experimental results obtained using the quantum computer show violation of all Mermin inequalities, with a clear degradation of the results in the five-qubit case. Though this quantum computer is not competitive to test Mermin inequalities as compared to other techniques when applied to a few qubits, it does offer the opportunity to explore multipartite entanglement for four and five qubits beyond the reach of other alternative technologies.
\end{abstract}

DOI: 10.1103/PhysRevA.94.012314

Quantum physics can be discriminated from classical physics using Bell-type inequalities [1]. In particular, the violation of Bell inequalities for two qubits has been extensively verified since they were first checked in atomic physics experiments [2,3]. Later on, the improvement of quantum optics techniques as well as other technologies such as nitrogen-vacancy (NV) centers has made it possible to eliminate many of the loopholes in the experimental verification of two-qubit Bell inequalities [3].

An extension of Bell inequalities to a larger number of particles corresponds to the set of Mermin inequalities [4]. Such inequalities should be maximally violated by GreenbergerHorne-Zeilinger (GHZ)-type states [5]. The experimental verification of multipartite Mermin inequalities faces the problem of a good control of three or more qubits, including the generation of entangled states, and the possibility of performing different measurements on each one. Violation of Mermin inequalities has been reported for three qubits [6] and four qubits [7], where all qubits are made out of photons, and for up to 14 qubits with a quantum computer based on ion traps [8].

In the case of superconducting qubits, violation of the Clauser-Horne-Shimony-Holt (CHSH) inequality was achieved in Ref. [9], whereas the GHZ construction and the three-qubit Mermin inequality violation was demonstrated in Ref. [10]. For a general review of theoretical and experimental progress in Bell inequalities, see Ref. [11].

The construction of the first prototypes of quantum computers allows for the possibility of experimenting with quantum states containing more than two qubits. In particular, IBM has opened the use of its five-qubit quantum computer to the community [12]. We here report results on the use of this quantum computer to test the violation of Mermin inequalitites for three, four, and five superconducting qubits.

\section{MERMIN POLYNOMIALS}

Local realism can be tested using Mermin polynomials. The technique to generate them is explained for example in Ref. [13]. The Mermin polynomial for three qubits is

$$
M_{3}=\left(a_{1} a_{2} a_{3}^{\prime}+a_{1} a_{2}^{\prime} a_{3}+a_{1}^{\prime} a_{2} a_{3}\right)-\left(a_{1}^{\prime} a_{2}^{\prime} a_{3}^{\prime}\right),
$$

where $a_{i}$ and $a_{i}^{\prime}$ correspond to two different settings for the measurement of each qubit $i$. Each measurement can take the values $\{-1,1\}$. Classical theories obey local realism (LR) which translates into a bound for the expectation value of the Mermin polynomial, $\left\langle M_{3}\right\rangle^{\mathrm{LR}} \leqslant 2$. Instead, for quantum mechanics (QM) the observables $a_{i}$ and $a_{i}^{\prime}$ are built out of linear combinations of Pauli matrices. Each measurement is expressed as a Kronecker product of the three local measurements and the expectation value for $\left\langle M_{3}\right\rangle$ is the maximum eigenvalue of the resulting $8 \times 8$ matrix. In this case, the maximum possible eigenvalue, and therefore the quantum bound, is $\left\langle M_{3}\right\rangle^{\mathrm{QM}} \leqslant 4$. We briefly construct circuits to check the violation of the classical bound on this inequality.

The Mermin polynomial that will be experimentally checked for four-qubits is

$$
\begin{aligned}
M_{4}= & -\left(a_{1} a_{2} a_{3} a_{4}\right)+\left(a_{1} a_{2} a_{3} a_{4}^{\prime}+a_{1} a_{2} a_{3}^{\prime} a_{4}+a_{1} a_{2}^{\prime} a_{3} a_{4}\right. \\
& \left.+a_{1}^{\prime} a_{2} a_{3} a_{4}\right)+\left(a_{1} a_{2} a_{3}^{\prime} a_{4}^{\prime}+a_{1} a_{2}^{\prime} a_{3} a_{4}^{\prime}+a_{1} a_{2}^{\prime} a_{3}^{\prime} a_{4}\right. \\
& \left.+a_{1}^{\prime} a_{2} a_{3} a_{4}^{\prime}+a_{1}^{\prime} a_{2} a_{3}^{\prime} a_{4}+a_{1}^{\prime} a_{2}^{\prime} a_{3} a_{4}\right)-\left(a_{1} a_{2}^{\prime} a_{3}^{\prime} a_{4}^{\prime}\right. \\
& \left.+a_{1}^{\prime} a_{2} a_{3}^{\prime} a_{4}^{\prime}+a_{1}^{\prime} a_{2}^{\prime} a_{3} a_{4}^{\prime}+a_{1}^{\prime} a_{2}^{\prime} a_{3}^{\prime} a_{4}\right)-\left(a_{1}^{\prime} a_{2}^{\prime} a_{3}^{\prime} a_{4}^{\prime}\right),
\end{aligned}
$$

with a classical bound of $\left\langle M_{4}\right\rangle^{\mathrm{LR}} \leqslant 4$ and a quantum bound of $\left\langle M_{4}\right\rangle^{\mathrm{QM}} \leqslant 8 \sqrt{2}$.

In the five-qubit case, the Mermin polynomial reads

$$
\begin{aligned}
M_{5}= & -\left(a_{1} a_{2} a_{3} a_{4} a_{5}\right)+\left(a_{1} a_{2} a_{3} a_{4}^{\prime} a_{5}^{\prime}+a_{1} a_{2} a_{3}^{\prime} a_{4} a_{5}^{\prime}\right. \\
& +a_{1} a_{2}^{\prime} a_{3} a_{4} a_{5}^{\prime}+a_{1}^{\prime} a_{2} a_{3} a_{4} a_{5}^{\prime}+a_{1} a_{2} a_{3}^{\prime} a_{4}^{\prime} a_{5} \\
& +a_{1} a_{2}^{\prime} a_{3} a_{4}^{\prime} a_{5}+a_{1}^{\prime} a_{2} a_{3} a_{4}^{\prime} a_{5}+a_{1} a_{2}^{\prime} a_{3}^{\prime} a_{4} a_{5} \\
& \left.+a_{1}^{\prime} a_{2} a_{3}^{\prime} a_{4} a_{5}+a_{1}^{\prime} a_{2}^{\prime} a_{3} a_{4} a_{5}\right) \\
& -\left(a_{1} a_{2}^{\prime} a_{3}^{\prime} a_{4}^{\prime} a_{5}^{\prime}+a_{1}^{\prime} a_{2} a_{3}^{\prime} a_{4}^{\prime} a_{5}^{\prime}+a_{1}^{\prime} a_{2}^{\prime} a_{3} a_{4}^{\prime} a_{5}^{\prime}\right. \\
& \left.+a_{1}^{\prime} a_{2}^{\prime} a_{3}^{\prime} a_{4} a_{5}^{\prime}+a_{1}^{\prime} a_{2}^{\prime} a_{3}^{\prime} a_{4}^{\prime} a_{5}\right),
\end{aligned}
$$

with a classical bound of $\left\langle M_{5}\right\rangle^{\mathrm{LR}} \leqslant 4$ and a quantum bound of $\left\langle M_{5}\right\rangle^{\mathrm{QM}} \leqslant 16$.

\section{CIRCUIT IMPLEMENTATION}

There are a number of technical issues associated with the specific implementation of the IBM five-qubit quantum computer. This quantum computer is based on superconducting flux qubits that live on a fridge with a temperature of about $15 \mathrm{mK}$, where only one of the qubits can be used to act as 

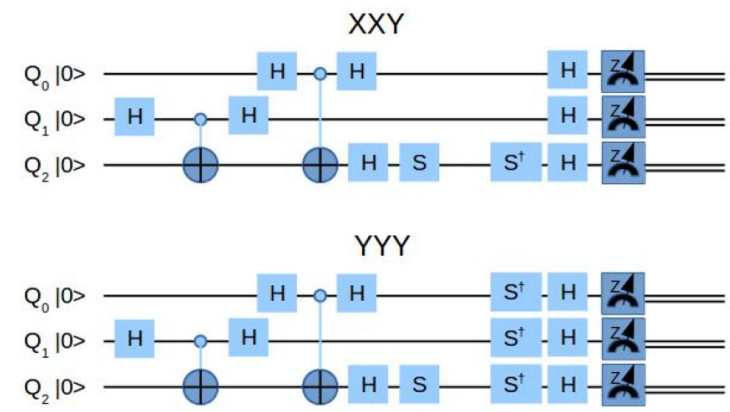

FIG. 1. The two circuits used for the three-qubit Mermin inequality. The first circuit corresponds to the $\sigma_{x} \sigma_{x} \sigma_{y}$ experiment, and the second circuit corresponds to the $\sigma_{y} \sigma_{y} \sigma_{y}$ experiment. The $S^{\dagger}$ gates make the difference between a $\sigma_{x}$ measurement and a $\sigma_{y}$ measurement.

the target qubit of any controlled-NOT (CNOT) gate. In the test of Mermin inequalitites, only GHZ-like states have to be created. This requires the use of a Hadamard gate on a control qubit followed by CNOTs targeted to the rest. In order to implement this kind of action we need to operate CNOT gates targeted to other qubits. This can be done using the relation $\mathrm{CNOT}_{1 \rightarrow 2}=\left(H_{1} \otimes H_{2}\right) \mathrm{CNOT}_{2 \rightarrow 1}\left(H_{1} \otimes H_{2}\right)$, where $H_{1}$ and $\mathrm{H}_{2}$ are Hadamard gates on qubits 1 and 2 , whereas $\mathrm{CNOT}_{1 \rightarrow 2}$ is the controlled-NOT gate which is controlled by qubit 1 .

In our choice of settings, the needed GHZ-like states have relative phases, as in the case of three qubits, where $|\phi\rangle=$ $1 / \sqrt{2}(|000\rangle+i|111\rangle)$. These phases are implemented using $S$ and $T$ gates, which are one-qubit gates that mutiply the $|1\rangle$ term with $\pi / 2$ and $\pi / 4$ phases, respectively. Measurements can only be done on the $\sigma_{z}$ basis, but they can be simulated in another basis with the help of additional gates, namely an $\mathrm{H}$ gate for $\sigma_{x}$ and an $S^{\dagger}$ gate followed by an $\mathrm{H}$ gate for $\sigma_{y}$.

Another relevant issue to be considered is that not all of the qubits are equally robust in the present quantum computer, some have relaxation and decoherence times larger than others, although all of them are of the order of $T=O(100 \mu \mathrm{s})$. We adapt our circuits to minimize the number of gates on the qubits that behave more poorly. For example, gates that implement phases that can be put freely in any qubit are allocated to the most robust ones.

Figures 1 and 2 represent the three circuits for the three-, four-, and five-qubit Mermin inequalities. In principle we need to perform as many experiments as the number of terms in the Mermin inequalities (1), (2), and (3). However due to our limited access to the computer and the symmetry of particle exchange of the states and the inequalities, only one experiment for a term representative of each number of primes $\left(a_{i}^{\prime}\right)$ was run. In our choice of settings, the number of primes amounts to the number of $\sigma_{y}$ measurements, whereas the nonprimes $\left(a_{i}\right)$ correspond to $\sigma_{x}$ measurements. We thus have two experiments for three qubits, five experiments for four qubits, and three experiments for five qubits. Each experiment was run 8192 times, the maximum available, except for the three-qubit experiments, which were run only 1024 times. When computing the expected value of the whole polynomial, each experiment was given the corresponding weight. In the errors discussion we compare results obtained when using the
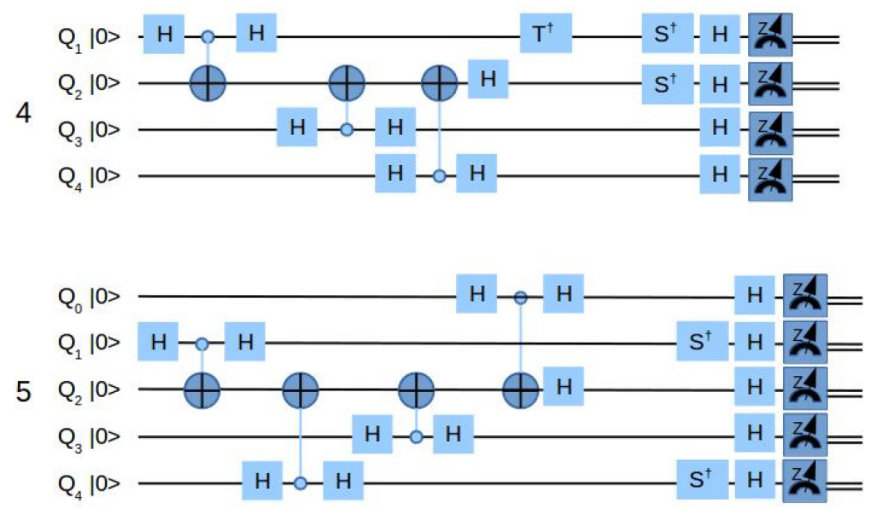

FIG. 2. Two of the circuits used for the four-qubit and five-qubit Mermin inequalities. The first circuit corresponds to the $\sigma_{y} \sigma_{y} \sigma_{x} \sigma_{x}$ experiment, whereas the second corresponds to the $\sigma_{x} \sigma_{y} \sigma_{x} \sigma_{x} \sigma_{y}$ experiment. The $S^{\dagger}$ gates make the difference between a $\sigma_{x}$ measurement and a $\sigma_{y}$ measurement. In order to change from $\sigma_{x}$ to $\sigma_{y}$, one has to add an $S^{\dagger}$ gate, or remove it to do the opposite. With this technique one can obtain all the circuits needed to test the inequalities.

symmetry with results obtained without using it, computing all the terms, for the three-qubit case.

\section{RESULTS}

We now give a more detailed discussion of the results for the three-qubit case and an abridged one for the four- and five-qubit cases, as much of it is basically the same.

In order to check the violation of the inequality, one has to choose the settings and the corresponding state that maximally violate it. One possibility is to choose the settings $a_{i}=\sigma_{x}$ and $a_{i}^{\prime}=\sigma_{y}$ for all the qubits. The state that maximizes the quantum violation in this case is $|\phi\rangle=1 / \sqrt{2}(|000\rangle+i|111\rangle)$.

The three-qubit Mermin inequality has four terms as shown in Eq. (1). In principle, four different circuits are needed, one for each term. The state will be the same for all of them, but the settings change. However, one can use the symmetry of the state and the inequality to reduce the number of measurements needed if there is limited access to the experimental setting as is our case. All the terms that have the same number of primes $\left(a_{i}^{\prime}\right)$ are represented by the same circuit by symmetry. We then considered only two different experiments, with 1024 runs each, the $\sigma_{x} \sigma_{x} \sigma_{y}$ experiment and the $\sigma_{y} \sigma_{y} \sigma_{y}$ experiment. The results are shown in Table I.

TABLE I. Table of detailed results for the two three-qubit experiments. In bold are results of even parity; in italic are results of odd parity. Counts for each result are expressed in probabilities computed out of 1024 runs. Computation of the expected value of $X X Y$ gives $\langle X X Y\rangle=0.715$ and of $Y Y Y$ gives $\langle Y Y Y\rangle=-0.710$. The combination $3\langle X X Y\rangle-\langle Y Y Y\rangle$ gives $\left\langle M_{3}\right\rangle_{\exp }=2.85 \pm 0.02$.

$\begin{array}{lllllllll}\text { Result } X X Y & \mathbf{0 0 0} & 001 & 010 & \mathbf{0 1 1} & 100 & \mathbf{1 0 1} & \mathbf{1 1 0} & 111\end{array}$ $\begin{array}{lllllllll}\text { Probability } & 0.229 & 0.042 & 0.024 & 0.194 & 0.043 & 0.203 & 0.231 & 0.033\end{array}$

$\begin{array}{lllllllll}\text { Result } Y Y Y & \mathbf{0 0 0} & 001 & 010 & \mathbf{0 1 1} & 100 & \mathbf{1 0 1} & \mathbf{1 1 0} & 111\end{array}$ $\begin{array}{lllllllll}\text { Probability } & 0.050 & 0.188 & 0.188 & 0.028 & 0.258 & 0.026 & 0.041 & 0.221\end{array}$ 
TABLE II. Table of results. LR corresponds to the local realism bound for each Mermin inequality, QM corresponds to the quantum bound, and Expt. is the experimental result.

\begin{tabular}{lccc}
\hline \hline & LR & QM & Expt. \\
\hline Three qubits & 2 & 4 & $2.85 \pm 0.02$ \\
Four qubits & 4 & $8 \sqrt{2}$ & $4.81 \pm 0.06$ \\
Five qubits & 4 & 16 & $4.05 \pm 0.06$ \\
\hline \hline
\end{tabular}

Eight probabilities for each term were obtained. In order to translate these probabilities to the expected values that appear in the inequality, one has to arrange the results in two groups according to the parity of the number of 1 (which represents the value -1 .) The expected value of the term is obtained by summing all the probabilities of the results of even parity and subtracting the results of odd parity. The correctly weighted sum of the expected values of each term gives the final result $\left\langle M_{3}\right\rangle_{\exp }=2.85 \pm 0.02$.

In the case of four qubits, the use of the settings $a_{i}=\sigma_{x}$ and $a_{i}^{\prime}=\sigma_{y}$ implies that the state that maximizes the quantum violation is $|\phi\rangle=1 / \sqrt{2}\left(e^{i \pi / 4}|0000\rangle+|1111\rangle\right)$. With these settings and this state, five experiments were performed, one for each term with different numbers of primes (2), with 8192 runs for each experiment. A result of $\left\langle M_{4}\right\rangle_{\exp }=4.81 \pm 0.06$ was obtained.

In the case of five qubits, the use of the settings $a_{i}=\sigma_{x}$ and $a_{i}^{\prime}=\sigma_{y}$ implies that the state that maximizes the quantum violation is $|\phi\rangle=1 / \sqrt{2}(|00000\rangle+|11111\rangle)$. With these settings and this state, three experiments were performed, one for each term with different numbers of primes (3), with 8192 runs for each experiment. A result of $\left\langle M_{5}\right\rangle_{\exp }=4.05 \pm 0.06$ was obtained. This is clearly a poor violation, which is still compatible with local realism. Improvement of the quantum computer is needed to obtain more accurate results, a summary of the results is presented in Table II.

The results obtained from the IBM quantum computer are subject to different kinds of errors. The stability of the quantum computer is still poor and the same experiments run at different times provided results that differed more than the expected behavior of statistical fluctuations. As an example, one month after the original runs, the three-qubit experiment was run again to compare results. This time, a result of $\left\langle M_{3}\right\rangle_{\exp }=$ $2.57 \pm 0.02$ was obtained, clearly showing the previous point. An additional run was done computing separately the four terms of Eq. (1), without assuming any symmetry, and a similar result was obtained, $\left\langle M_{3}\right\rangle_{\exp }=2.57 \pm 0.02$, showing that it is safe to assume the symmetry of parity exchange.
We may get an estimation of the statistical error as a dispersion around the mean. We may, as well, treat the results as a multinomial distribution, using the expression $\delta p=$ $\sqrt{p(1-p) / N}$, which for $N=8192$ gives $\delta p=O\left(10^{-2}\right)$. The different Mermin inequalities for three, four, and five qubits require a different number of experiments to be done, which are considered as independent. We may then add in quadrature its errors, which is the figure we associate with the explicit results. In this sense, the five-qubit result obtained with the present quantum computer does not have sufficient statistical significance to discard local realism.

Furthermore, some of the issues related to the elimination of loopholes cannot be addressed. Experiments suffer from errors related to stability, loss of coherence, and lack of full fidelity of the quantum gates. This is clearly seen as the violation of Mermin inequalities deteriorate progressively as the numbers of qubits, and gates used in the experiment, increase. We may think of the experimental verification of Mermin inequalitites as a test of the overall fidelity of the whole Mermin circuit.

\section{CONCLUSIONS}

Experimental verification of Mermin inequalities for three, four, and five qubits has been tested on a five-qubit IBM quantum computer. Results do show violation of local realism in all cases, with a clear degradation in quality as the number of qubits (and needed gates) increases. Nonetheless, this produces the first experimental violation of four- and five-qubit Mermin inequalities with superconducting qubits, though the statistical significance of the second one is still poor. It should be noted however that, in the case of the four-qubit inequality, the result shows generic nonlocality but does not provide evidence for genuine four-particle nonlocality, because this would only be implied by $M_{4}>8$. [14]. It can be argued that the measurements of Mermin polynomials for many qubits can be used as a figure of merit to assess the fidelity of a quantum computer.

\section{ACKNOWLEDGMENTS}

D.A. acknowledges financial help from the APIF Scholarship of the University of Barcelona. J.I.L. acknowledges financial support from Grant No. FIS2013-41757-P. We acknowledge use of the IBM Quantum Experience for this work.

The views expressed are those of the authors and do not reflect the official policy or position of IBM or the IBM Quantum Experience team.
[1] J. S. Bell, Physics 1, 195 (1964).

[2] A. Aspect, J. Dalibard, and G. Roger, Phys. Rev. Lett. 49, 1804 (1982).

[3] B. Hensen et al., Nature (London) 526, 682 (2015); L. K. Shalm et al., Phys. Rev. Lett. 115, 250402 (2015); B. Hensenet al., arXiv:1603.05705.

[4] N. D. Mermin, Phys. Rev. Lett. 65, 1838 (1990).
[5] R. F. Werner and M. M. Wolf, Phys. Rev. A 64, 032112 (2001).

[6] J. W. Pan et al., Nature (London) 403, 515 (2000); C. Erwen et al., Nat. Photonics 8, 292 (2014).

[7] Z. Zhao, T. Yang, Y. A. Chen, A. N. Zhang, M. Zukowski, and J. W. Pan, Phys. Rev. Lett. 91, 180401 (2003).

[8] B. P. Lanyon, M. Zwerger, P. Jurcevic, C. Hempel, W. Dur, H. J. Briegel, R. Blatt, and C. F. Roos, Phys. Rev. Lett. 112, 100403 (2014). 
[9] M. Ansmann et al., Nature (London) 461, 504 (2009).

[10] L. Di Carlo et al., Nature (London) 467, 574 (2010); M. Neeley et al., ibid. 467, 570 (2010).

[11] N. Brunner, D. Cavalcanti, S. Pironio, V. Scarani, and S. Wehner, Rev. Mod. Phys. 86, 419 (2014).
[12] IBM Quantum Experience, http://www.research.ibm.com/ quantum.

[13] D. Alsina, A. Cervera, D. Goyeneche, J. I. Latorre, and K. Zyczkowski, arXiv:1606.01991.

[14] D. Collins, N. Gisin, S. Popescu, D. Roberts, and V. Scarani, Phys. Rev. Lett. 88, 170405 (2002). 